\title{
Ethnic disparities in postpartum hemorrhage after cesarean delivery: a retrospective case-control study
}

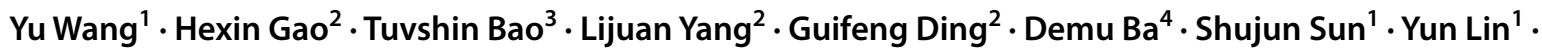 \\ Shanglong $\mathrm{Yao}^{1}{ }^{\mathbb{D}}$
}

Received: 23 December 2019 / Accepted: 8 January 2021 / Published online: 28 January 2021

(c) The Author(s) 2021

\begin{abstract}
Purpose To explore the relationship of ethnicity and postpartum hemorrhage (PPH) for women who underwent cesarean delivery (CD) and examine the risk factors for PPH in distinct ethnic groups in China.

Methods We conducted case-control studies with the maternity data from the 11,778 CD cases, in Xinjiang Uygur Autonomous Region. Initially, multivariable logistic regression was used to estimate the disparity of race-ethnicity on the risk of PPH in ethnic Han, Uygur, Hui and Kazakh. Then, we performed case-control studies within two major ethnic groups, identifying the specific risk factors for PPH.

Results Ethnic Uygur were associated with a statistically significant increased odds [adjusted odds ratios (aOR) 2.05; 95\% confidence interval (CI) 1.26-3.33] of PPH compared with ethnic Han. For subgroup analyses, in Uygur subgroup, general anesthesia (aOR 7.78; 95\% CI 2.31-26.20); placenta previa (aOR 11.18; 95\% CI 3.09-40.45); prenatal anemia (aOR 4.84; 95\% CI 2.44-9.60); emergency surgery (aOR 4.22; 95\% CI 1.95-9.13) were independently associated with PPH. In Han subgroup, general anesthesia (aOR 5.70; 95\% CI 1.89-17.26); placenta previa (aOR 20.08; 95\% CI 6.35-63.46); multiple pregnancy (aOR 7.21; 95\% CI 1.61-32.37); body mass index (aOR 1.19; 95\% CI 1.07-1.31) were the risk factors to PPH. Conclusion Uygur have more tendency to PPH compared to Han, and risk factors for PPH in Uygur and Han groups may differ. Knowing these differences may be meaningful when planning interventions and resources for high-risk patients undergoing cesarean delivery, and we need more research aimed at risk factors for PPH.
\end{abstract}

Keywords Postpartum hemorrhage $\cdot$ Ethnicity $\cdot$ Obstetric anesthesia

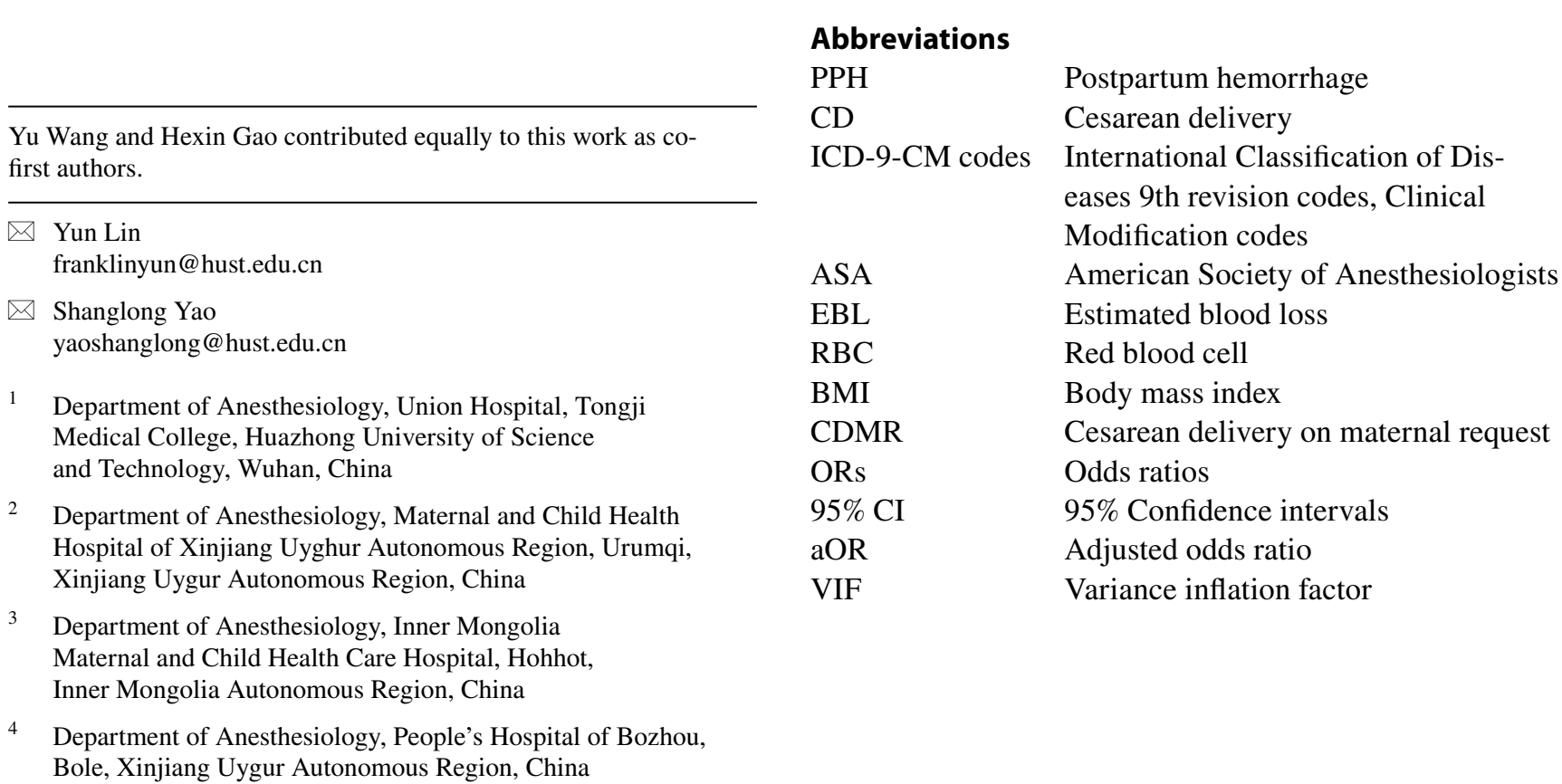




\section{Introduction}

Postpartum hemorrhage (PPH) is a severe complication that may result in adverse outcomes of perinatal mothers and infants. Worldwide, a woman dies due to massive PPH approximately every 4 min [1]. PPH is the major cause of maternal mortality in China which accounted for $28 \%$ of all maternal deaths in 2013 [2]. It is also an important cause of pregnancy-related morbidity, such as multi-organ failure and peripartum hysterectomy $[1,3]$. There are a variety of etiologies of PPH, including uterine atony, retained placenta, lacerations of the birth canal, uterine rupture, placenta accreta, various types of coagulopathies, uterine inversion and infection, each of which has diverse risk factors $[4,5]$. The major cause of PPH is uterine atony, accounting for approximately $80 \%$ of PPH cases, and often occurs in the absence of recognized risk factors [6]. A number of changes after implementation of the two-child policy in 2016 in obstetric practice and maternal demographics in China may have contributed to an increased rate of $\mathrm{PPH}$, including an increasing rate of cesarean delivery (CD) and more pregnant women of advanced maternal age [7]. It is therefore necessary to determine risk factors for PPH to plan interventions and resources for high-risk patients.

Racial-ethnic disparities are persistent problems in pregnant women's health and obstetric outcomes. In some Western countries, race as an independent risk factor for PPH has been documented $[8,9]$. Some retrospective studies indicated racial and ethnic disparities in PPH in the US $[8,9]$. $\mathrm{CD}$ increases blood loss at delivery and thus is a risk factor of PPH [5]. However, few previous study did pay specific attention to women who underwent $\mathrm{CD}$. Moreover, to date, there are no studies assessing the racial-ethnic disparities in PPH among ethnic groups in China, because of the limited data of PPH among different ethnic groups in the same setting and the same period. Xinjiang Uygur Autonomous Region (referred to as Xinjiang in the remainder of this study) has a large multi-ethnic population with 47 ethnic components, and Urumqi is the capital city of Xinjiang. The ethnic group with the largest population is ethnic Han, the other ethnic groups mainly include ethnic Uygur, Hui and Kazakh. A unique data set from different ethnic groups in Xinjiang Region, accumulates large amounts of cases undergoing CD and suffering from PPH to examine risk factors.

In this study, we conducted a retrospective case-control study to test the hypothesis that the ethnicity is an independent risk factor for PPH. It was further determined whether the individual risk factors for PPH vary among women who underwent $C D$ in the two largest ethnicity subgroups.

\section{Methods}

\section{Ethics approval and consent to participate}

After obtaining Ethics Committee of the Xinjiang Uygur Autonomous Region Maternal and Child Health Hospital approval on 31 January 2018 (Ethical approval number: XJFYLL2018001), we registered our project at the Chinese clinical trial registry (Registration number: ChiCTR1800014752). We had no access to information that could identify individual participants, so our research does not involve patients' personal information and written informed consent was waived.

\section{Study designs}

We performed retrospective analyses with case-control study design to explore risk factors for PPH among ethnic Han, Uygur, Hui and Kazakh in Xinjiang Region. The cases were the $\mathrm{CD}$ women diagnosed as PPH and the controls were identified from the CD parturients without PPH selected with matched factors, and the ratio of the case:control was 1:2. Maternal age and gestational weeks were considered as the matching factors because advanced maternal age (age $>35)[10,11]$ and preterm births [12] are known risk factors. Premature labor was defined when gestational weeks $<37$ compared with reference group defined as gestational weeks $\geq 37$.

\section{Data resources}

Data were collected from parturients who underwent CD in the Xinjiang Uygur Autonomous Region Maternal and Child Health Hospital, from January 2014 to January 2017. We extracted information from Electronic Medical Record, Laboratory Information System, Picture Archiving and Communication Systems, and Anesthesia Information System. For each parturient, the following information is extracted and classified: (1) demographic characteristics; (2) obstetric characteristics; (3) comorbidities; (4) fetal conditions; (5) clinical managements. The diagnoses and procedures were recorded using International Classification of Diseases 9th Revision, Clinical Modification codes (ICD-9-CM codes) [13].

\section{Inclusion criteria and exclusion criteria}

Our study only screened women who underwent CD. We included the parturients of ethnic Han, Uygur, Hui and Kazakh, because these are the major ethnic groups that make up the largest proportion. The inclusion criteria were 
the women who underwent $\mathrm{CD}$; women aged 16 years and older; patients categorized as American Society of Anesthesiologists (ASA) I-III; information of the hospital discharge records was detailed. After the first-round screening, we excluded women from the study for the following reasons: the patients with uncertain or missing hospital records (such as predelivery hemoglobin undetermined or past medical history unrecorded); patients with antenatal bleeding or bleeding owing to preoperative thrombocytopenia and coagulation factor decreased; patients categorized as ASA IV; patients who converted from spinal anesthesia to general anesthesia.

\section{Primary outcomes and potential confounders}

The primary outcome was PPH defined as an estimated blood loss (EBL) $\geq 1000 \mathrm{~mL}$ after CD according to ICD9-CM codes, or red blood cell (RBC) transfusion, both within $24 \mathrm{~h}$ [14]. The blood loss was measured based on the amount of fluid in the aspirator and the weight of the dressings, as well as estimated by the surgeons (subjective estimated blood loss). ICD-9-CM codes for PPH are wellverified, showing a positive predictive value of $80 \%$ [15]. As blood loss may often be underestimated by clinicians $[16,17], \mathrm{RBC}$ transfusion is a component in the definition of severe PPH [18]. The primary independent variable for this study was ethnicity. We classified ethnicities into four groups: Han, Uygur, Hui and Kazakh, with Han as the reference group. Previous studies [6, 8, 9, 19-24] indicated potential confounders and we divided those variables into 5 categories: (1) demographic characteristics; (2) obstetric characteristics; (3) comorbidities; (4) fetal conditions; (5) clinical managements. The demographic characteristics contained maternal age [19], racial-ethnicity [8, 9], body mass index (BMI) [20, 21], maternal educational level. Gestational weeks, gravidity, parity, number of previous $\mathrm{CD}$, and previous uterine scar were categorized as obstetric characteristics. Relevant maternal comorbidities and fetal conditions were identified using corresponding ICD-9-CM codes and included diabetes, hypertension, gynecological tumor, prenatal anemia, placental abruption, placenta previa, stillbirth, multiple pregnancy, cephalopelvic disproportion, malpresentation, and macrosomia. Clinical managements contained emergency surgery, number of antenatal visits, assisted conception, intrapartum $\mathrm{CD}$, cesarean delivery on maternal request (CDMR), anesthesia methods [23, 24] included general anesthesia and spinal anesthesia.

\section{Statistical methods}

In the primary analysis, we identified women with PPH and matched controls among the four ethnic populations. The univariate analyses were performed to generate crude odds ratios (ORs) with 95\% confidence intervals $(95 \% \mathrm{CI})$. We then fitted multivariable logistic regression models to determine the relationship between the ethnicity and the PPH to generate adjusted ORs (aOR), with adjustments for all variables that $P$ value $<0.05$ in the univariate analyses. In the stratified analyses, we performed separate analyses among women in ethnic Han and Uygur undergoing CD, with univariable and multivariable logistic regression models.

Before each logistic regression model, restricted cubic spline functions were used to test whether the associations met the assumptions of a linear relationship for each continuous variable associated with PPH. We categorized continuous variables if they were not linearly related to PPH or they could be categorized by clinically relevant cut points (predelivery hemoglobin was categorized and cut by $100 \mathrm{~g} / \mathrm{L}$ to represent the prenatal anemia). Gravidity, parity, number of previous $\mathrm{CD}$, number of antenatal visits, maternal educational level and BMI were regarded as continuous variables. The effect of potential collinearity on the estimates for ethnicity was assessed by calculating a variance inflation factor (VIF) between candidate variables. Collinearity was determined to be insignificant if VIF scores $<10$. The Hosmer-Lemeshow statistics were calculated for each model to evaluate the goodness of models' fit. $P$ value $<0.05$ was considered statistically significant and all tests were two-tailed. Demographic and clinical characteristics were presented by frequency, proportion, mean, and standard deviation. All statistical analyses were carried out using the SAS 9.4 (SAS Institute, Cary, NC, USA).

\section{Results}

A total of 11,778 CD cases were included in our study. Most pregnant women were ethnic Han women (43.7\%) and Uygur women (39.2\%), followed by Hui (9.8\%) and Kazakh (7.3\%) women. There were 244 cases identified as PPH finally. Among PPH cases, 166 (68.0\%) women had at least $1000 \mathrm{~mL}$ EBL, and 147 (60.2\%) women received RBC transfusion intraoperatively or within $24 \mathrm{~h}$ after $\mathrm{CD}$. The overall rate of PPH was $2.1 \%$ (95\% CI 1.8-2.3\%). Subject enrollment and analysis are illustrated in Fig. 1.

In the primary analysis of the four ethnic populations, we identified 244 women with PPH and 488 matched controls. Characteristics of cases and controls were presented in Table 1. Of the 24 potential confounders in Table 1, there were 14 variables with $P$ value $<0.05$ in the univariate analyses, included ethnicity, general anesthesia, placenta previa, placental abruption, multiple pregnancy, previous uterine scar, gravidity, number of previous $\mathrm{CD}$, number of antenatal visits, maternal educational level, diabetes, still birth, prenatal anemia, emergency surgery. Unadjusted and adjusted odds ratios (aOR) for ethnicities in multivariable 


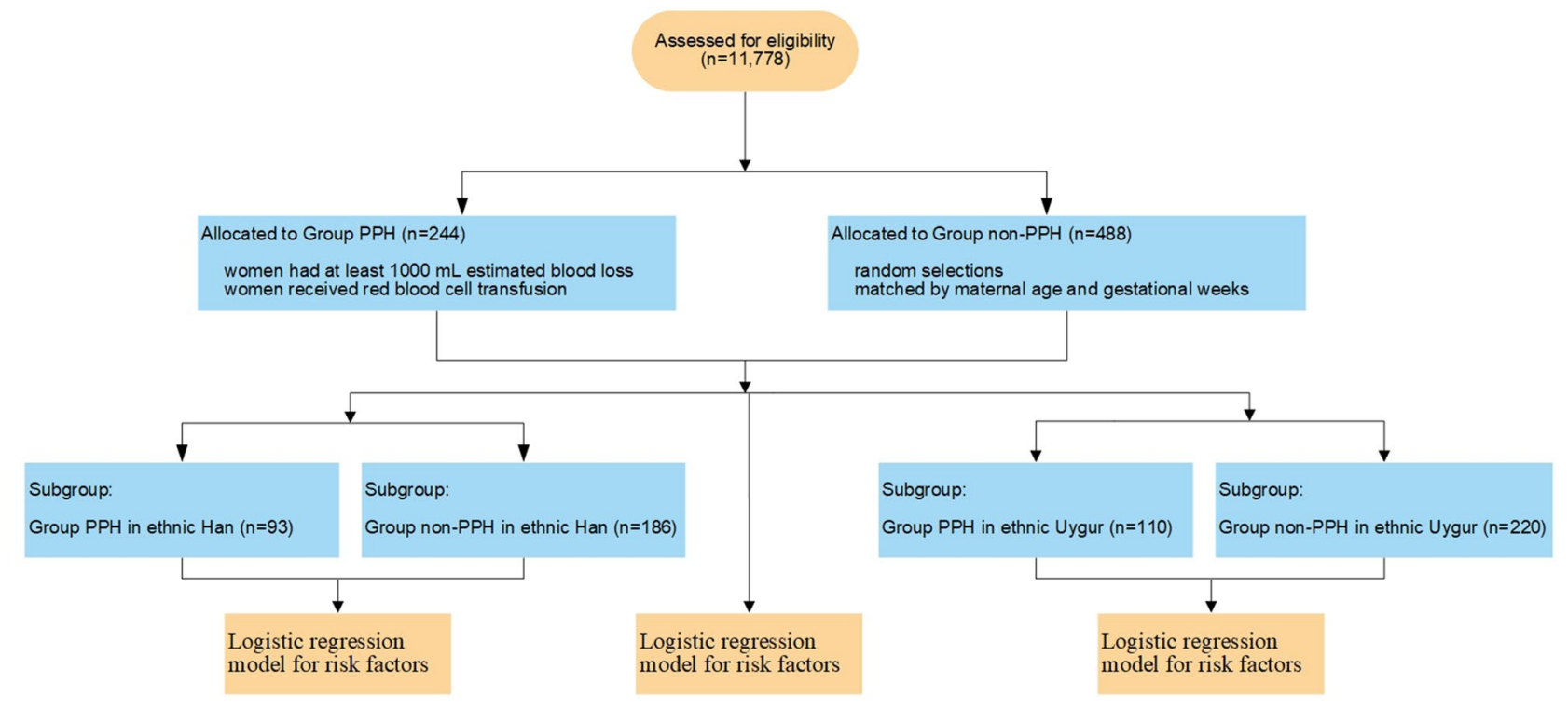

Fig. 1 Subject enrollment and analysis flowchart

analysis were showed in Table 2. After the adjustment for potential mediators, ethnic Uygur and Kazakh were independently associated with PPH, with a statistically significant increased odds of PPH (aOR 2.05; 95\% CI 1.26-3.33) and (aOR 3.83; 95\% CI 1.80-8.16) in comparison with Han. The $P$ value for Hosmer-Lemeshow test was $0.07>0.05$, indicating good fit of the model.

In stratified analyses, we identified 110 cases in ethnic Uygur and 93 cases in ethnic Han. The matched controls were selected randomly in the Han and Uygur parturients, respectively, with the ratio of the case:control $=1: 2$. Characteristics of women who are Han or Uygur with and without PPH were presented in Table 3.

In the Uygur groups, there were 13 statistically significant independent variables, included maternal educational level, gravidity, parity, number of previous CD, scared uterus, prenatal anemia, placental abruption, placenta previa, stillbirth, emergency surgery, number of antenatal visits, intrapartum $C D$, general anesthesia. In the Han cohort, the 11 variables with $P$ value $<0.05$ in the univariate analyses were BMI, gravidity, parity, number of previous CD, scared uterus, diabetes, placenta previa, multiple pregnancy, macrosomia, number of antenatal visits, general anesthesia. Unadjusted and adjusted odds ratios for possible risk factors selected for multivariable analysis were showed in Tables 4 and 5 for Uygur and Han, respectively. For ethnic Uygur subgroup, placenta previa (aOR 11.18; 95\% CI 3.09-40.45), general anesthesia (aOR 7.78; 95\% CI 2.31-26.20; reference group = spinal anesthesia), prenatal anemia (aOR 4.84; 95\% CI 2.44-9.60) and emergency surgery (aOR 4.22; 95\% CI 1.95-9.13) were independently associated with PPH. For ethnic Han subgroup, placenta previa (aOR 20.08; 95\% CI 6.35-63.46) and general anesthesia (aOR 5.70; 95\% CI 1.89-17.26; reference group $=$ spinal anesthesia) were also more likely to $\mathrm{PPH}$. Other risk factors for ethnic Han were BMI (aOR 1.19; 95\% CI 1.07-1.31) and multiple pregnancy (aOR 7.21; 95\% CI 1.61-32.37). The Hosmer-Lemeshow test for the models was $P$ value 0.87 (Uygur group) and 0.47 (Han group), indicating that the models had modest ability to discriminate patients with or without PPH.

\section{Discussion}

Our study confirmed that ethnic Uygur is a risk factor for PPH compared with ethnic Han in CD cohort. In different ethnic populations, general anesthesia and placenta previa were common risk factors for $\mathrm{PPH}$ and placenta previa was the factor with the highest risk. For women undergoing CD with general anesthesia, the Uygur were more likely to have PPH. Prenatal anemia and emergency surgery were risk factors for Uygur while BMI and multiple pregnancy were risk factors for Han.

PPH has been the leading cause of maternal mortality in China and worldwide [5, 25, 26]. While risk factors for PPH have been extensively studied, little is known regarding racial-ethnicity disparities in $\mathrm{PPH}$, particularly in China. Among previous studies worldwide documenting whether racial-ethnicity disparities exist with regard to $\mathrm{PPH}$, one study was conducted in vaginal birth [27] and the other three studies were not able to adequately adjust for potential confounding factors (e.g. maternal education level, age, BMI, 
Table 1 Characteristics of included parturients with or without postpartum hemorrhage

\begin{tabular}{|c|c|c|}
\hline Independent variables & PPH (244) & Control (488) \\
\hline \multicolumn{3}{|l|}{ Race/ethnicity } \\
\hline Han & $93(38.1 \%)$ & $247(50.6 \%)$ \\
\hline Uygur & $110(45.1 \%)$ & $147(30.1 \%)$ \\
\hline Hui & $18(7.4 \%)$ & $60(12.3 \%)$ \\
\hline Kazakh & $23(9.4 \%)$ & $34(7.0 \%)$ \\
\hline BMI (weight/height ${ }^{2}$ ) & $29.0 \pm 3.6$ & $28.3 \pm 3.5$ \\
\hline Maternal educational level & $2.4 \pm 0.7$ & $2.6 \pm 0.6$ \\
\hline Gravidity & $2.3 \pm 0.8$ & $1.9 \pm 0.8$ \\
\hline Parity & $1.9 \pm 0.7$ & $1.7 \pm 0.7$ \\
\hline Times of CD & $1.6 \pm 0.7$ & $1.3 \pm 0.5$ \\
\hline Scared uterus & $131(53.7 \%)$ & $159(32.6 \%)$ \\
\hline Diabetes & $41(16.8 \%)$ & $41(8.4 \%)$ \\
\hline Hypertension & $12(4.9 \%)$ & $10(2.0 \%)$ \\
\hline Gynecological tumor $^{1}$ & $6(2.5 \%)$ & $9(1.8 \%)$ \\
\hline Prenatal aknemia & $95(38.9 \%)$ & $117(24.0 \%)$ \\
\hline Placental abruption & $21(8.6 \%)$ & $7(1.4 \%)$ \\
\hline Placenta previa & $73(29.9 \%)$ & $17(3.5 \%)$ \\
\hline Multiple pregnancy & $17(7.0 \%)$ & $4(0.8 \%)$ \\
\hline Cephalopelvic disproportion & $2(0.8 \%)$ & $4(0.8 \%)$ \\
\hline Macrosomia & $112(45.9 \%)$ & $213(43.6 \%)$ \\
\hline Malpresentation $^{2}$ & $28(11.5 \%)$ & $73(15.0 \%)$ \\
\hline Still birth & $15(6.2 \%)$ & $5(1.0 \%)$ \\
\hline Emergency surgery & $163(66.8 \%)$ & $208(42.6 \%)$ \\
\hline Number of antenatal visits & $3.6 \pm 3.9$ & $5.8 \pm 4.3$ \\
\hline Assisted conception & $5(2.1 \%)$ & $11(2.3 \%)$ \\
\hline Intrapartum CD & $27(11.1 \%)$ & $68(13.9 \%)$ \\
\hline CDMR & $4(1.6 \%)$ & $25(5.1 \%)$ \\
\hline \multicolumn{3}{|l|}{ Anesthesia method } \\
\hline Spinal anesthesia & $189(77.5 \%)$ & $470(96.3 \%)$ \\
\hline General anesthesia & $55(22.5 \%)$ & $18(3.7 \%)$ \\
\hline
\end{tabular}

Data presented as $n(\%)$; mean $\pm \mathrm{SD}$

$P P H$ postpartum hemorrage, $B M I$ body mass index, $C D$ cesarean delivery, $C D M R$ cesarean delivery on maternal request

${ }^{1}$ Gynecological tumor: such as pregnancy with cervical cancer, giant cervical fibroids, subuterine fibroids, etc.

${ }^{2}$ Malpresentation: fetal transverse position, first-born full-term single breech (estimated fetal birth weight $>3500 \mathrm{~g}$ ) and foot first exposure

macrosomia) [8, 9, 28]. Moreover, no study has analyzed such association in specific ethnic cohorts.

The causes of PPH include uterine contraction, genital tract trauma, placental factors, damage and abnormal blood coagulation. Various factors influence each other, and PPH is often the result of several factors. Though the uterine atony occurs in only $5 \%$ of labor, approximately $80 \%$ of PPH cases result from the uterine atony [6]. However, the uterine atony can take place secretively with no predictive factors. It may result from an inadequate response to endogenous and exogenous signals [8]. Many PPH cases often occur from uterine
Table 2 Unadjusted and adjusted odds ratios for ethnicity associated with postpartum hemorrhage

\begin{tabular}{llll}
\hline Race/ethnicity & $\begin{array}{l}\text { Unadjusted Odds } \\
\text { Ratio }^{1}\end{array}$ & $\begin{array}{l}\text { Adjusted Odds } \\
\text { Ratio }^{2}\end{array}$ & $P$ value $^{3}$ \\
\hline Han & Reference & Reference & Reference \\
Uygur & $1.99(1.41-2.80)$ & $2.05(1.26-3.33)$ & 0.004 \\
Hui & $0.80(0.45-1.42)$ & $1.27(0.61-2.65)$ & 0.520 \\
Kazakh & $1.80(1.01-3.21)$ & $3.83(1.80-8.16)$ & 0.001 \\
\hline
\end{tabular}

${ }^{1} \mathrm{~A}$ total of 244 cases of postpartum hemorrhage in four ethnic groups were identified, and 488 matched controls. Crude odds ratios (ORs) of ethnicity related to postpartum hemorrhage were calculated, with their $95 \%$ confidence intervals $(95 \% \mathrm{CI})$

${ }^{2}$ Adjusted odds ratios (ORs) of ethnicity related to postpartum hemorrhage were calculated, with their $95 \%$ confidence intervals $(95 \%$ CI) by multivariable logistic regression included all variables with $P<0.05$ in the univariable analyses

${ }^{3}$ The $P$ value represents the statistical significance of ethnicity in the multivariable model

atony do not have identifiable antepartum risk factors [6]. Therefore, we should not only pay attention to the high-risk factors, but also monitor and intervene in women with hidden risk factors like ethnicities. Ethnic disparities in pregnant women's health and obstetric outcomes may mainly result from social-economical factors, including language barriers, healthcare resources, cultural barriers, and genetic differences. Ethnic-specific risk factors, such as multiple pregnancy and high BMI in ethnic Han and prenatal anemia and emergency surgery in Uygur, were found in our study.

Anesthesia is necessary for women undergoing $\mathrm{CD}$ and whether general anesthesia or spinal anesthesia is a risk factor for PPH is worth studying [24]. Previous studies have shown that, in general anesthesia, many drugs could suppress the contraction of animal and human uterine muscles, like intravenous general anesthetics which include propofol, midazolam, ketamine and opioids [29], volatile anesthetics which include sevoflurane and desflurane [30]. On the other hand, general anesthetics might suppress platelet function and hemostasis. Sevoflurane is demonstrated to alter bleeding time in a reversible and dose-related manner [31]. The intravenous anesthetic propofol may inhibit platelet aggregation [32]. Potential causes coming from the general anesthetics should be discussed. However, we need to notice that general anesthesia or intubation is a part of protocol for critical postpartum complications so the anesthesiologists may prefer to use general anesthesia for patients with obstetric complications. Previous study by Butwick et al. [24] has conducted the sensitivity analyses for the pre-labor cohort. They excluded the conditions that general anesthesia may be considered more often for women with obstetric complications including placenta previa or abnormal placentation. The results showed that the relationship between general anesthesia and severe PPH still persisted, albeit with wider 
Table 3 Characteristics in parturients of ethnic Han and Uygur with or without postpartum hemorrhage

\begin{tabular}{|c|c|c|c|c|}
\hline \multirow[t]{2}{*}{ Independent variables } & \multicolumn{2}{|l|}{ Han } & \multicolumn{2}{|l|}{ Uygur } \\
\hline & $\mathrm{PPH}(n=93)$ & Control $(n=186)$ & $\mathrm{PPH}(n=110)$ & Control $(n=220)$ \\
\hline BMI (weight/height ${ }^{2}$ ) & $29.5 \pm 3.6$ & $28.2 \pm 3.1$ & $28.8 \pm 3.6$ & $29.7 \pm 4.5$ \\
\hline Maternal educational level & $2.5 \pm 0.6$ & $2.6 \pm 0.6$ & $2.3 \pm 0.7$ & $2.6 \pm 0.6$ \\
\hline Gravidity & $2.2 \pm 0.8$ & $1.8 \pm 0.8$ & $2.4 \pm 0.7$ & $1.8 \pm 0.8$ \\
\hline Parity & $1.6 \pm 0.7$ & $1.4 \pm 0.6$ & $2.2 \pm 0.7$ & $1.5 \pm 0.7$ \\
\hline Number of previous CD & $1.5 \pm 0.6$ & $1.2 \pm 0.5$ & $1.8 \pm 0.7$ & $1.3 \pm 0.6$ \\
\hline Previous uterine scar & $43(46.2 \%)$ & $44(23.7 \%)$ & $71(64.5 \%)$ & $63(28.6 \%)$ \\
\hline Diabetes & $21(22.6 \%)$ & $17(9.1 \%)$ & $14(12.7 \%)$ & $25(11.4 \%)$ \\
\hline Hypertension & $5(5.4 \%)$ & $3(1.6 \%)$ & $6(5.5 \%)$ & $10(4.5 \%)$ \\
\hline Gynecological tumor $^{1}$ & $4(4.3 \%)$ & $2(1.1 \%)$ & 0 & $2(0.9 \%)$ \\
\hline Prenatal anemia & $18(19.4 \%)$ & $29(15.6 \%)$ & $60(54.5 \%)$ & $33(15.0 \%)$ \\
\hline Placental abruption & $4(4.3 \%)$ & $3(1.6 \%)$ & $13(11.8 \%)$ & $4(1.8 \%)$ \\
\hline Placenta previa & $29(31.2 \%)$ & $5(2.7 \%)$ & $32(29.1 \%)$ & $5(2.3 \%)$ \\
\hline Multiple pregnancy & $8(8.6 \%)$ & $4(2.2 \%)$ & $9(8.2 \%)$ & $13(5.9 \%)$ \\
\hline Cephalopelvic disproportion & $1(1.1 \%)$ & $1(0.5 \%)$ & $1(0.9 \%)$ & $2(0.9 \%)$ \\
\hline Macrosomia & $23(24.7 \%)$ & $27(14.5 \%)$ & $16(14.5 \%)$ & $38(17.3 \%)$ \\
\hline Malpresentation $^{2}$ & $10(10.8 \%)$ & $24(12.9 \%)$ & $14(12.7 \%)$ & $33(15 \%)$ \\
\hline Stillbirth & $3(3.2 \%)$ & 0 & $8(7.3 \%)$ & $1(0.5 \%)$ \\
\hline Emergency surgery & $58(62.4 \%)$ & $130(69.9 \%)$ & $87(79.1 \%)$ & $118(53.6 \%)$ \\
\hline Number of antenatal visits & $4.3 \pm 4.0$ & $6.3 \pm 4.3$ & $2.6 \pm 3.2$ & $4.0 \pm 3.8$ \\
\hline Assisted conception & $5(5.4 \%)$ & $2(1.1 \%)$ & 0 & $3(1.4 \%)$ \\
\hline Intrapartum CD & $13(14.0 \%)$ & $27(14.5 \%)$ & $7(6.4 \%)$ & $34(15.5 \%)$ \\
\hline CDMR & $1(1.1 \%)$ & $5(2.7 \%)$ & $2(1.8 \%)$ & $4(1.8 \%)$ \\
\hline \multicolumn{5}{|l|}{ Anesthesia method } \\
\hline Spinal anesthesia & $79(84.9 \%)$ & $177(95.2 \%)$ & $77(70.0 \%)$ & $214(97.3 \%)$ \\
\hline General anesthesia & $14(15.1 \%)$ & $9(4.8 \%)$ & $33(30.0 \%)$ & $6(2.7 \%)$ \\
\hline
\end{tabular}

Data presented as $n(\%)$; mean $\pm \mathrm{SD}$

$P P H$ postpartum hemorrage, $B M I$ body mass index, $C D$ cesarean delivery, $C D M R$ cesarean delivery on maternal request

${ }^{1}$ Gynecological tumor: such as pregnancy with cervical cancer, giant cervical fibroids, subuterine fibroids, etc.

${ }^{2}$ Malpresentation: fetal transverse position, first-born full-term single breech (estimated fetal birth weight $>3500 \mathrm{~g}$ ) and foot first exposure confidence intervals. Nevertheless, the relationship between PPH and general anesthesia should be considered in cautions, and further studies are in need.

\section{Strengths and limitations}

The large number of births in the Xinjiang Uygur Autonomous Region Maternal and Child Health Hospital accumulates sufficient cases undergoing $\mathrm{CD}$ and suffering from $\mathrm{PPH}$ to examine risk factors among different ethnic groups. We incorporated various aspects of covariates categorized as demographic characteristics, obstetric characteristics and comorbidities, fetal conditions and clinical management. Particularly, we were able to examine the role of anesthesia methods and emergency $\mathrm{CD}$ in the association between ethnicity and $\mathrm{PPH}$, which were rarely addressed by previous studies. By robustly adjusting the role of comprehensive potential covariates of the association of ethnicity and PPH, we made valid conclusions.

Our study, however, had a number of limitations. The sample size of control group was small compared with 11,534 non-PPH subjects which we sampled from. Potential selection bias may affect the results, even though we used two matched factors to control the selection bias. Despite our efforts to adjust for a full range of candidate variables, some unmeasured factors, such as maternal socioeconomic status, prolonged third-stage labor [33], vertical incision, preeclampsia and uterine incision [34], were not captured in our analyses. We did not collect data for the parturients by vaginal delivery because the $\mathrm{CD}$ has potential risks related to numerous complications and it is a cause of PPH [5], besides, $\mathrm{CD}$ rate is elevating rapidly worldwide [35]. 
Table 4 Identification of predictive factors of postpartum hemorrhage among Uygur subgroup
Table 5 Identification of predictive factors of postpartum hemorrhage among Han subgroup

\begin{tabular}{lccc}
\hline Risk factors & Unadjusted odds ratio $^{1}$ & Adjusted odds ratio $^{2}$ & $P$ value $^{3}$ \\
\hline Maternal educational level & $0.53(0.38-0.74)$ & $0.53(0.38-0.74)$ & 0.63 \\
Gravidity & $2.85(2.09-3.89)$ & $1.33(0.68-2.59)$ & 0.41 \\
Parity & $3.71(2.63-5.24)$ & $1.67(0.73-3.80)$ & 0.22 \\
Number of previous CD & $2.84(2.00-4.03)$ & $1.38(0.51-3.79)$ & 0.53 \\
Previous uterine scar & $4.54(2.79-7.39)$ & $2.33(0.59-9.19)$ & 0.23 \\
Prenatal anemia & $6.80(4.01-11.52)$ & $\mathbf{4 . 8 4}(\mathbf{2 . 4 4 - 9 . 6 0 )}$ & $\mathbf{0 . 0 0}$ \\
Placental abruption & $7.24(2.30-22.76)$ & $1.50(0.28-8.15)$ & 0.64 \\
Placenta previa & $17.64(6.64-46.89)$ & $\mathbf{1 1 . 1 8}(\mathbf{3 . 0 9}-\mathbf{4 0 . 4 5})$ & $\mathbf{0 . 0 0}$ \\
Stillbirth & $17.18(2.12-139.16)$ & $2.33(0.21-25.70)$ & 0.49 \\
Emergency surgery & $3.27(1.92-5.56)$ & $\mathbf{4 . 2 2}(\mathbf{1 . 9 5}-\mathbf{9 . 1 3})$ & $\mathbf{0 . 0 0}$ \\
Number of antenatal visits & $0.89(0.83-0.96)$ & $1.04(0.95-1.14)$ & 0.38 \\
Intrapartum CD & $0.37(0.16-0.87)$ & $1.08(0.35-3.31)$ & 0.89 \\
General anesthesia & $15.29(6.17-37.90)$ & $\mathbf{7 . 7 8}(\mathbf{2 . 3 1 - 2 6 . 2 0})$ & $\mathbf{0 . 0 0}$ \\
\hline
\end{tabular}

$P P H$ postpartum hemorrhage, $C D$ cesarean delivery

${ }^{1}$ Statistically significant associations in the univariable model are presented

${ }^{2}$ Statistically significant associations in the multivariable model are denoted by bold text and only variables with $P$ value $<0.05$ comes into multivariable model after univariable logistic regression

${ }^{3}$ The $P$ value represents the statistical significance of all factors in the multivariable model

\begin{tabular}{|c|c|c|c|}
\hline Risk factors & Unadjusted odds ratio $^{1}$ & Adjusted odds ratio ${ }^{2}$ & $P$ value $^{3}$ \\
\hline BMI (weight/height ${ }^{2}$ ) & $1.13(1.04-1.22)$ & $1.19(1.07-1.31)$ & $\mathbf{0 . 0 0}$ \\
\hline Gravidity & $1.76(1.30-2.39)$ & $1.17(0.74-1.86)$ & 0.50 \\
\hline Parity & $2.09(1.40-3.12)$ & $0.95(0.42-2.15)$ & 0.90 \\
\hline Number of previous CD & $2.59(1.60-4.17)$ & $1.16(0.31-4.28)$ & 0.83 \\
\hline Previous uterine scar & $2.78(1.63-4.71)$ & $2.57(0.63-10.51)$ & 0.19 \\
\hline Diabets & $2.90(1.45-5.82)$ & $1.88(0.81-4.38)$ & 0.14 \\
\hline Placenta previa & $16.40(6.09-44.19)$ & $20.08(6.35-63.46)$ & $\mathbf{0 . 0 0}$ \\
\hline Multiple pregnancy & $4.28(1.26-14.62)$ & $7.21(1.61-32.37)$ & 0.01 \\
\hline Macrosomia & $1.94(1.04-3.61)$ & $1.43(0.63-3.21)$ & 0.39 \\
\hline Number of antenatal visits & $0.89(0.84-0.95)$ & $0.94(0.87-1.01)$ & 0.08 \\
\hline General anesthesia & $3.49(1.45-8.39)$ & $5.70(1.89-17.26)$ & 0.00 \\
\hline
\end{tabular}

$P P H$ postpartum hemorrhage, $B M I$ body mass index, $C D$ cesarean delivery

${ }^{1}$ Statistically significant associations in the univariable model are presented

${ }^{2}$ Statistically significant associations in the multivariable model are denoted by bold text and only variables with $P$ value $<0.05$ comes into multivariable model after univariable logistic regression

${ }^{3}$ The $P$ value represents the statistical significance of all factors in the multivariable model

\section{Conclusion}

The current study confirmed that maternal ethnicity is an independent risk factor for PPH and provided insights into the risk factors for PPH in different ethnicities. Hence, it may be important to stratify women by ethnicities for prevention, intervention, and treatment of the PPH. By augmenting access to health education, services and opportune perinatal care in the specific ethnic, the risk of PPH may reduce. Such analyses would require population-wide studies using nuanced clinical data. The epidemiological results will also be conducive to identify future research fields aimed at risk factors for PPH.

Acknowledgements We would like to thank Dr. Peng Yin from Chinese Center for Disease Control and Prevention for his comments and help with writing in English. We also acknowledge the researchers of Xinjiang Uygur Autonomous Region Maternal and Child Health Hospital for the assistance of the data collection.

Author contributions YW, HG contributed equally to these work of the acquisition, analysis, or interpretation of data and drafted the 
manuscript; LY carried out data collection; GD carried out patients screening; DB and TB contributed to data analysis; YL and SY contributed equally to conceiving the idea of the manuscript, revising the manuscript critically for important intellectual content and final approval of the version to be published.

Funding This research did not receive any specific grant from funding agencies in the public, commercial, or not-for-profit sectors.

Availability of data and materials The datasets used and/or analysed during the current study are available from the corresponding author on reasonable request.

\section{Compliance with ethical standards}

Conflict of interest The authors declare that they have no conflict of interest.

Open Access This article is licensed under a Creative Commons Attribution 4.0 International License, which permits use, sharing, adaptation, distribution and reproduction in any medium or format, as long as you give appropriate credit to the original author(s) and the source, provide a link to the Creative Commons licence, and indicate if changes were made. The images or other third party material in this article are included in the article's Creative Commons licence, unless indicated otherwise in a credit line to the material. If material is not included in the article's Creative Commons licence and your intended use is not permitted by statutory regulation or exceeds the permitted use, you will need to obtain permission directly from the copyright holder. To view a copy of this licence, visit http://creativecommons.org/licenses/by/4.0/.

\section{References}

1. Sebghati M, Chandraharan E. An update on the risk factors for and management of obstetric haemorrhage. Women's Health (Lond Engl). 2017;13(2):34-40.

2. Wang X, Ding J, Wang L. Analysis of maternal mortality in Xinjiang from 2005 to 2013. Chin J Woman Child Health Res. 2018;1:35-8.

3. McDonald S. Management of the third stage of labor. J Midwifery Women's Health. 2007;52(3):254-61.

4. Simpson KR. Update on evaluation, prevention, and management of postpartum hemorrhage. MCN. Am J Matern Child Nurs. 2018;43(2): 120 .

5. Oyelese Y, Ananth CV. Postpartum hemorrhage: epidemiology, risk factors, and causes. Clin Obstet Gynecol. 2010;53(1):147-56.

6. Bateman BT, Berman MF, Riley LE, Leffert LR. The epidemiology of postpartum hemorrhage in a large, nationwide sample of deliveries. Anesth Analg. 2010;110(5):1368-73.

7. Zhang HX, Zhao YY, Wang YQ. Analysis of the characteristics of pregnancy and delivery before and after implementation of the twochild policy. Chin Med J (Engl). 2018;131:37-42.

8. Bryant A, Mhyre JM, Leffert LR, Hoban RA, Yakoob MY, Bateman BT. The association of maternal race and ethnicity and the risk of postpartum hemorrhage. Anesth Analg. 2012;115(5):1127-36.

9. Harvey SA, Lim E, Gandhi KR, Miyamura J, Nakagawa K. racialethnic disparities in postpartum hemorrhage in Native Hawaiians, Pacific Islanders, and Asians. Hawaii J Med Public Health. 2017;76(5):128-32.

10. Oakley L, Penn N, Pipi M, Oteng-Ntim E, Doyle P. Risk of adverse obstetric and neonatal outcomes by maternal age: quantifying individual and population level risk using routine UK maternity data. PLoS ONE. 2016;11(10):e164462.
11. Kramer MS, Berg C, Abenhaim H, Dahhou M, Rouleau J, Mehrabadi $\mathrm{A}$, Joseph $\mathrm{KS}$. Incidence, risk factors, and temporal trends in severe postpartum hemorrhage. Am J Obstet Gynecol. 2013;209:441-9.

12. Sheldon WR, Blum J, Vogel JP, Souza JP, Gulmezoglu AM, Winikoff B. Postpartum haemorrhage management, risks, and maternal outcomes: findings from the World Health Organization Multicountry Survey on Maternal and Newborn Health. BJOG-Int J Obstet Gynaecol. 2014;121(Suppl 1):5-13.

13. Goff SL, Pekow PS, Markenson G, Knee A, Chasan-Taber L, Lindenauer PK. Validity of using ICD-9-CM codes to identify selected categories of obstetric complications, procedures and co-morbidities. Paediatr Perinat Epidemiol. 2012;26:421-9.

14. Newsome J, Martin JG, Bercu Z, Shah J, Shekhani H, Peters G. Postpartum hemorrhage. Tech Vasc Interv Radiol. 2017;20(4):266-73.

15. Lain SJ, Roberts CL, Hadfield RM, Bell JC, Morris JM. How accurate is the reporting of obstetric haemorrhage in hospital discharge data? A validation study. Aust N Z J Obstet Gyn. 2008;48(5):481-4.

16. Prata N, Gerdts C. Measurement of postpartum blood loss. BMJ. 2010;340:c555.

17. Rath WH. Postpartum hemorrhage-update on problems of definitions and diagnosis. Acta Obstet Gyn Scan. 2011;90(5):421-8.

18. Deneux-Tharaux C, Dupont C, Colin C, Rabilloud M, Touzet S, Lansac J, Harvey T, Tessier V, Chauleur C, Pennehouat G, Morin $\mathrm{X}$, Bouvier-Colle MH, Rudigoz R. Multifaceted intervention to decrease the rate of severe postpartum haemorrhage: the PITHAGORE6 cluster-randomised controlled trial. BJOG-Int J Obstet Gy. 2010;117(10):1278-87.

19. Lao TT, Sahota DS, Cheng YKY, Law LW, Leung TY. Advanced maternal age and postpartum hemorrhage-risk factor or red herring? J Matern-Fetal Neonata Med. 2014;27(3):243-6.

20. Paglia MJ, Grotegut CA, Johnson LNC, Thames B, James AH. Body mass index and severe postpartum hemorrhage. Gynecol Obstet Investig. 2012;73(1):70-4.

21. Blomberg M. Maternal obesity and risk of postpartum hemorrhage. Obstet Gynecol. 2011;118(3):561-8.

22. Skjeldestad FE, Oian P. Blood loss after cesarean delivery: a registry-based study in Norway, 1999-2008. Am J Obstet Gynecol. 2012;206(1):71-6.

23. Chang CC, Wang IT, Chen YH, Lin HC. Anesthetic management as a risk factor for postpartum hemorrhage after cesarean deliveries. Am J Obstet Gynecol. 2011;205(5):461-2.

24. Butwick AJ, Ramachandran B, Hegde P, Riley ET, El-Sayed YY, Nelson LM. Risk factors for severe postpartum hemorrhage after cesarean delivery: case-control studies. Anesth Analg. 2017;125(2):523-32.

25. Say L, Chou D, Gemmill A, Tuncalp O, Moller A, Daniels J, Gulmezoglu AM, Temmerman M, Alkema L. Global causes of maternal death: a WHO systematic analysis. Lancet Glob Health. 2014;2(6):e323-33.

26. Hogan MC, Foreman KJ, Naghavi M, Ahn SY, Wang M, Makela SM, Lopez AD, Lozano R, Murray CJL. Maternal mortality for 181 countries, 1980-2008: a systematic analysis of progress towards Millennium Development Goal 5. Lancet. 2010;375(9726):1609-23.

27. Combs CA, Murphy EL, Laros RKJ. Factors associated with postpartum hemorrhage with vaginal birth. Obstet Gynecol. 1991;77(1):69-76.

28. Guendelman S, Thornton D, Gould J, Hosang N. Obstetric complications during labor and delivery: assessing ethnic differences in California. WHI. 2006;16(4):189-97.

29. Thind AS, Turner RJ. In vitro effects of propofol on gravid human myometrium. Anaesth Intensive Care. 2008;36(6):802-6.

30. Yoo KY, Lee JC, Yoon MH, Shin M, Kim SJ, Kim YH, Song TB, Lee J. The effects of volatile anesthetics on spontaneous contractility of isolated human pregnant uterine muscle: a comparison among 
sevoflurane, desflurane, isoflurane, and halothane. Anesth Analg. 2006;103(2):443-7.

31. Yokubol B, Hirakata H, Nakamura K, Sai S, Okuda H, Hatano Y, Urabe N, Mori K. Anesthesia with sevoflurane, but not isoflurane, prolongs bleeding time in humans. J Anesth. 1999;13(4):193-6.

32. Hirakata H, Nakamura K, Yokubol B, Toda H, Hatano Y, Urabe N, Mori K. Propofol has both enhancing and suppressing effects on human platelet aggregation in vitro. Anesthesiology. 1999;91(5):1361-9.

33. Bais JMJ, Eskes M, Pel M, Bonsel GJ, Bleker OP. Postpartum haemorrhage in nulliparous women: incidence and risk factors in low and high risk women. A Dutch population-based cohort study on standard $(\geq 500 \mathrm{ml})$ and severe $(\geq 1000 \mathrm{ml})$ postpartum haemorrhage. Eur J Obstet Gyn R B. 2004;115(2):166-72.
34. Combs CA, Murphy EL, Laros RKJ. Factors associated with hemorrhage in cesarean deliveries. Obstet Gynecol. 1991;77(1):77-82.

35. Betran AP, Torloni MR, Zhang JJ, Gulmezoglu AM. WHO statement on caesarean section rates. BJOG-Int J Obstet Gy. 2016;123(5):667-70.

Publisher's Note Springer Nature remains neutral with regard to jurisdictional claims in published maps and institutional affiliations. 